\title{
Enfoque etnográfico y formación docente: aportes para el trabajo de enseñanza
}

\author{
Laura Cerletti*
}

\section{Resumen}

Este artículo aborda algunos aspectos centrales del enfoque etnográfico y su relevancia para pensar la formación docente. Se plantea su importancia para la comprensión en profundidad sobre los docentes como sujetos sociales, considerando especialmente sus experiencias -en su vinculación con los contextos sociohistóricos y las tramas de relaciones de los que forman parte-, a través de las que se va construyendo el propio lugar docente. Se recupera la importancia de esta dimensión en tanto abre el registro a una diversidad poco indagada. Tomando la categoría “familia" como analizador, se profundiza sobre los aportes del enfoque etnográfico a la formación para el trabajo de enseñanza, argumentando sobre la importancia de recuperar esa diversidad de experiencias vividas por los docentes para facilitar un proceso de construcción de debates, más que de definiciones cerradas, y como una modalidad de trabajo que permite una mayor problematización sobre la diversidad existente en el mundo social.

\section{Palabras clave}

etnografía; experiencias; docentes; diversidad; familia.
* Docente Cátedra Sinisi, Departamento de Ciencias Antropológicas (Facultad de Filosofía y Letras, UBA), Buenos Aires. Investigadora (categoría Asistente) en el Consejo Nacional de Investigaciones Científicas y Técnicas (Conicet), Argentina. laurabcerletti@yahoo.com.ar 


\title{
Ethnographic approach and teachers' training: contributions to the work of teaching
}

\begin{abstract}
This paper focuses on some central characteristics of the ethnographic approach and its relevance in reflections on teachers' training. It is here presented its importance for an indepth understanding ofteachers as social subjects, considering mainly their experiences - and their relationships with the social and historical contexts and networks they are part of, which build up their position as teachers. Thus, the importance of this dimension is pointed out since it makes possible the recording of a diversity that has not been extensively studied so far. Taking the category "family" as an analyzer, we specify the contributions of the ethnographic approach to teachers' training, arguing about the importance of considering this diversity of experiences lived by teachers. This may allow for a process of construction of debates, rather than of closed definitions, and a modality of work that enables a deeper understanding of the diversity present in the social world.
\end{abstract}

Key words ethnography; experiences; teachers; diversity; family. 


\section{Introducción}

El campo de la Antropología y la Educación lleva décadas de desarrollo, y ha logrado una importante consolidación en el contexto latinoamericano, en buena medida de la mano de los aportes de la etnografía educativa. Frente a los múltiples significados que ha adquirido esta última, es central señalar que con ella aludimos a algo que trasciende en mucho una mera cuestión de método o de técnicas de recolección de datos. Por enfoque etnográfico estamos entendiendo una concepción teórico-metodológica del mundo social, que recoge y rediscute aportes clásicos de la Antropología, en diálogo con contribuciones importantes de la historiografía (especialmente aquella que tematiza los procesos sociales recuperando la dimensión cotidiana y experiencial de los sujetos en las diversas formaciones históricas), entre otros aportes teóricos, según ha sido profundamente trabajado por algunos autores clave de este campo (ver Achilli, 2005; Rockwell, 2009; entre otros).

Sin embargo, es fundamental avanzar sobre una aclaración. Al decir de la investigadora mexicana Elsie Rockwell, “la etnografía no produce por sí misma una alternativa pedagógica” (2009, p. 26). Adelantando lo que vamos a desarrollar en los próximos apartados, acordamos enteramente con la autora cuando plantea que la contribución de la etnografía a los procesos de transformación educativa se vinculan con "la posibilidad de recuperar lo particular y lo significativo desde lo local, pero además, de situarlo en una escala social más amplia y en un marco conceptual más general” (2009, p. 34). Uno de los aspectos centrales en que se puede recuperar este nivel de lo local viene de la mano de valorar el saber docente, diferenciándolo (aunque por supuesto con intersecciones insoslayables) del discurso pedagógico, en tanto está centralmente constituido por las propias experiencias de los docentes a partir de sus prácticas cotidianas (Rockwell, 2009; ver también Achilli, 2000; Batallán, 2007, entre otros).

Este artículo busca recuperar la importancia de estos aportes para la formación docente $^{1}$, identificando algunos caminos para concretar y especificar estas contribuciones, tomando como analizador una categoría que también ha sido históricamente abordada por la Antropología: la "familia"; categoría a la que se alude con toda frecuencia desde el campo educativo (Nogueira, 2011; Cerletti, 2010), y que suele constituir asimismo un área presente en diversas formulaciones sobre la enseñanza de las Ciencias Sociales.

1. Por razones de espacio, no podemos hacer acá un estado de la cuestión exhaustivo sobre los trabajos que han realizado contribuciones sustantivas a esta temática, pero sí mencionar algunas producciones que nos resultan especialmente sugestivas al respecto (trabajando sobre distintas modalidades para potenciar esta articulación): Achilli, 2000; Batallán, 2007; Berteley Busquets, 2001; Edelstein, 2002; Rockwell, 2009; Sinisi et. al., 2011; entre otros. 


\section{Enfoque etnográfico, formación docente y experiencias}

Desde el enfoque que venimos planteando, se ha definido a la etnografía como un proceso de documentar lo no-documentado de la realidad social (Rockwell, 2009), en alusión a “lo cotidiano, lo oculto, lo inconsciente” (Rockwell, 2009, p. 21). En este sentido, la vida cotidiana no constituye una dimensión diferente (o por fuera de) la historia, sino justamente es “el ‘centro’ del acaecer histórico” (Heller, 1972, p. 42). La vida cotidiana está impregnada de contenidos históricos, y su análisis delimita y recupera conjuntos heterogéneos de actividades emprendidos y articulados por sujetos particulares (Ezpeleta y Rockwell, 1987). De tal forma, este enfoque hace su aporte entendiendo la existencia cotidiana como historia acumulada, abordando el presente en términos historizados (Neufeld, 1997) y relacionales (Achilli, 2005). Es decir, reconstruyendo analíticamente las relaciones existentes entre diversos procesos sociohistóricos y las acciones y significaciones registradas en el presente, que son siempre llevadas a cabo (construidas, tensionadas, disputadas) por los sujetos. Es así que estamos concibiendo a los sujetos como protagonistas de los procesos sociales a través de su accionar cotidiano, y por tanto, como conocedores prácticos e intérpretes polémicos de la realidad (Batallán y García, 1992). No ya entendidos “como individuos aislados sino en interacción/relación con otros que es el único modo de producción de sentidos en tanto no existe sujeto fuera de las relaciones sociales" (Achilli, 2005, p. 25). Esta recuperación del punto de vista de los sujetos (incluyendo sus prácticas) desde un enfoque relacional requiere a su vez un análisis que lo vincule y lo tensione con las condiciones objetivas del contexto sociohistórico del que forman parte, en su doble carácter de generador de limitaciones y posibilidades.

Así, con una concepción dialéctica de la relación entre sujeto y estructura, desde este enfoque se han discutido nociones centrales para el estudio de los procesos educativos tales como ‘socialización'. Superando aquellas conceptualizaciones que ponían el acento en la transmisión de la cultura y su asimilación (relativamente pasiva o automática) por parte de las nuevas generaciones, se han desarrollado trabajos claves en torno a la categoría de experiencias formativas ${ }^{2}$, que recupera el carácter activo y transformador de la agencia humana. Esta idea se aplica a sujetos de distintas edades; es decir, pensar los procesos formativos a través de los modos en que son vividos, experimentados, por los sujetos, dentro de las formaciones históricas que

2. Es central en este sentido mencionar los aportes fundantes de Elsie Rockwell y Elena Achilli. los constituyen (y que son a su vez constituidas por los mismos), alcanza tanto a los niños como 
a los adultos. Entendemos que éste es un planteo importante para la comprensión de la formación docente. Ésta se produce por supuesto en los contextos e instituciones especialmente (e intencionalmente) dedicados a ello, tales como los institutos de formación docente, profesorados, magisterios, cursos de capacitación, etc. Pero también las diversas experiencias vividas por los docentes son formativas (Rockwell, 1995), en sentidos múltiples y heterogéneos: es a partir de las mismas que se va construyendo el propio lugar docente y las modalidades de trabajo de enseñanza, siempre como parte de tramas de relaciones que incluyen a muchos otros sujetos ${ }^{3}$.

En este proceso, las experiencias tempranas de escolarización tienen una relevancia importante para comprender los modos en que se construyen las prácticas docentes (Alliaud, 2007; Goodson, 2003). Pero junto a las experiencias relativas a la escolarización, como dijimos en el párrafo anterior, el proceso formativo se va constituyendo a partir de múltiples experiencias, que pueden estar vinculadas tanto a la escolarización (en sus distintos niveles) como a otros aspectos de sus vidas (familiar, laboral, entre otros) (Cerletti, 2010). Y sin embargo, uno de los aspectos sobre los que se ha prestado poca atención desde el ámbito investigativo tiene que ver con las experiencias de los docentes más allá de su escolarización, es decir, no recortando de antemano otras dimensiones de sus vidas, también constitutivas de su trabajo.

Como venimos planteando, la comprensión de estos procesos formativos (y por tanto, de construcción de subjetividades) es inescindible de las configuraciones sociohistóricas de las que forman parte y en las que se constituyen. En este sentido, la categoría de experiencia es un articulador conceptual fuerte, en tanto permite vincular analíticamente la realidad social con los modos en que es incorporada y procesada por los sujetos (y al mismo tiempo, permanentemente producida). Al respecto, entendemos que facilita acceder a una distinción crítica entre "realidad (lo que está realmente ahí afuera, sea lo que ello fuera), experiencia (cómo esa realidad se presenta a sí misma a la conciencia) y las expresiones (cómo la experiencia individual es encuadrada y articulada)" (Bruner, 1986, p. 6). De tal forma, las expresiones de las experiencias (Bruner, 1986) son centrales para entender las subjetividades, ya que las mismas se ponen en juego complejamente en las prácticas de los sujetos, es decir, de modos que no son lineales ni causales 4 .

Esta argumentación surge de las conclusiones de una investigación etnográfica que incluyó un

3. Para una discusión en profundidad sobre la formación docente a partir de la noción de "práctica docente", así como su articulación con la investigación, se puede consultar Achilli, 2000.

4. Por esto es imposible establecer apriorísticamente qué dimensiones de las experiencias serán determinantes en los procesos de configuración de las subjetividades. 
eje de trabajo en torno a las experiencias formativas de maestros y maestras de escuela primaria. El tema general de la indagación giró en torno a las relaciones entre las familias y las escuelas en contextos de desigualdad social, buscando documentar sentidos y prácticas cotidianas puestos en juego por diversos adultos con niños en edad escolar a su cargo y por docentes, entre otros actores sociales, prestando especial atención a las propias experiencias y trayectorias de los sujetos con los que trabajamos. Desde lo registrado a lo largo del trabajo de campo, realizado en distintos períodos entre 2004 y 2008 en un barrio de la zona sur de la Ciudad de Buenos Aires ${ }^{5}$, y desde la concepción relacional del abordaje con el que se fue desarrollando, se puso en evidencia la variedad de experiencias a la que venimos aludiendo y su relevancia. Al abrir el registro y análisis a múltiples dimensiones, no determinadas a priori sino trabajadas a partir de la importancia atribuida por los propios sujetos, se pudo observar que las trayectorias de los docentes implican recorridos sumamente heterogéneos, eventualmente en una misma persona y entre las/os distintos/as maestros/as (Cerletti, 2010). Esto a su vez demuestra que la posición como maestra/ os se construye activamente, en procesos que pueden incluir contradicciones y paradojas, a través de la conjugación de diversas dimensiones de las experiencias vividas y de las posibilidades vinculadas a cada contexto sociohistórico (Cerletti, 2010), independientemente de que sean conscientemente puestas en uso en el presente, de modos más o menos explícitos, o no, a través de procesos que no necesariamente se

5. Se trata de una "ex villa de emergencia" que atravesó un proceso de regularización de la propiedad de los terrenos (mediante procesos de organización comunitaria) y de autoconstrucción de las viviendas. En su configuración actual se observan claras marcas de su historia como villa. En ese contexto, se mantuvieron contactos con varias instituciones, a partir de las cuales se establecieron vinculaciones con adultos con niños en edad escolar a su cargo, con los que desarrollamos entrevistas abiertas y observación participante en diversas situaciones cotidianas. También se llevaron adelante entrevistas en profundidad con docentes y observación participante en una escuela situada en el mismo barrio. Asimismo, esta indagación se complementó con el análisis de fuentes secundarias y documentales (leyes, normativas, actas, circulares, producciones de organizaciones barriales, publicaciones de organismos nacionales e internacionales, etc.), y con entrevistas con sujetos vinculados a distintas instituciones que atienden a niños y familias (Centro de Salud, Parroquia, Equipo de Orientación Escolar, entre otros). hacen manifiestos.

Ahora bien, ¿qué relevancia pueden tener estas experiencias para el trabajo de enseñanza? A continuación, avanzaremos sobre este interrogante, tomando un eje en particular, vinculado con la misma investigación, y que remite a una categoría especialmente significativa -de distintos modostanto para el campo de la Antropología como para la Educación (y sus intersecciones).

\section{El aporte de las experiencias: "Familia" como analizador}

El campo de lo que es significado y vivido como “familia”, al trabajar con las experiencias expresa- 
das por los sujetos, denota la existencia de una enorme diversidad. Sin embargo, paradojalmente, en las situaciones en que se enuncia cómo deben ser las familias, esa diversidad frecuentemente no es considerada, o es valorada negativamente. Concretamente, al hablar sobre las familias de sus alumnos ${ }^{6}$, es recurrente que los docentes las describan en términos negativos, especialmente en los casos en que ubican situaciones de monoparentalidad, o de crianza con adultos que no son los padres biológicos, o de parejas con una cantidad importante de hijos, por mencionar algunos. Hemos registrado que en muchas ocasiones estas formas familiares son caracterizadas como "mal constituidas", incluso como "no familias". Entendemos en este sentido que ejerce una fuerza considerable el peso representacional que tiene la llamada familia nuclear occidental (Segalen, 1992), como forma válida o correcta de ser “familia”. Efectivamente, al menos en la Argentina, estas representaciones de la familia nuclear como "la" familia, han sido también instaladas y reforzadas desde la misma escuela a lo largo de los años. Y si bien contemporáneamente se vienen observando indicios de modificaciones respecto a esta forma hegemónica de representar a "la" familia", no deja aun de estar presente como un modelo. Aquello que se aleja del mismo, sería considerado como "deficitario", y como causante de límites e imposibilidades en el pleno desarrollo de los niños, y por lo tanto, de su escolarización (Cerletti, 2006).

La paradoja interesante resulta del hecho de que muchas veces los mismos docentes que expresan estas representaciones, al contar sus propias experiencias, evidencian formas de constitución y organización familiar dinámicas, cambiantes, y con suma frecuencia, también alejadas de este modelo. Y esta diversidad de las propias experiencias no suele ser vivida como habiendo obturado la escolarización o el pleno desarrollo de la persona. De hecho, sus propios procesos de escolarización se llevaron a cabo junto con -y como parte de-esta diversidad (Cerletti, 2010).

Sin embargo, algunos de los docentes con los que trabajamos sí hacían un uso explícito de sus propias experiencias para enriquecer y reflexionar sobre su trabajo de enseñanza. $Y$ es en este punto en el que nos interesa retomar más específicamente el interrogante planteado más arriba, sobre la relevancia de estas experiencias para el trabajo de enseñanza y el enriquecimiento de la formación docente.

6. Estas consideraciones surgen tanto de la investigación mencionada anteriormente, como de una investigación anterior que se centró más específicamente sobre las representaciones de los docentes respecto a las familias de sus alumnos. El trabajo de campo en ese caso se llevó a cabo en dos escuelas de otra zona de la Ciudad de Buenos Aires (ver Cerletti, 2006).

7. En el año 2010 en la Argentina se promulgó (no sin fuertes resistencias de algunos sectores sociales) la llamada Ley de Matrimonio Igualitario que genera la posibilidad de ampliar las formas legitimadas de ser familia, al equiparar en derechos y obligaciones a las familias encabezadas por parejas del mismo sexo. 
Como explicitamos en el apartado anterior, todo ese cúmulo diverso y heterogéneo de experiencias vividas por los sujetos en sus vidas cotidianas es mucho más que anecdótico, o simplemente reproducción rutinaria de la vida. Se trata de modos de hacer (al decir de Michel De Certeau, 1996), constitutivos de lo social, y que al mismo tiempo implican huellas de procesos históricos, complejamente configurados en el presente. Las experiencias expresadas por nuestros entrevistados respecto a sus historias familiares permiten ir entretejiendo este entramado de procesos históricos en los cuales las situaciones económicas, las cuestiones laborales, los vaivenes políticos de nuestra historia, tuvieron un lugar más o menos explicitado, pero que efectivamente posibilitan la reconstrucción analítica de los márgenes de posibilidades y limitaciones de cada tiempo histórico, dentro de las cuales los sujetos fueron también construyendo sus propias preferencias y recorridos. Por tanto, las experiencias vividas, en su heterogeneidad, generan un potencial muy interesante de saberes y conocimientos pasibles de incorporarse al trabajo en las aulas.

¿Cómo trabajarlas? ¿Cómo buscar formas de hacer que trasciendan el mero relato personal, que puede o no querer ser compartido con los estudiantes? Pensamos que no se trata de que los docentes expongan sus vidas en su trabajo de enseñanza, pero sí nos parece importante puntualizar los aportes que mencionamos al principio, que se pueden hacer desde el campo de la Antropología, y de la etnografía más específicamente, para conceptualizarlas de modo que posibiliten niveles más profundos de trabajo en las escuelas con la diversidad realmente existente.

En el eje específico que trajimos -“la familia”-, es importante mencionar que ha sido uno de los objetos clásicos de estudio dentro del campo de la Antropología. Durante muchos años, se la concibió como una institución universal que, con particularidades locales, satisfacía necesidades básicas de la reproducción humana. Sin embargo, esto ha sido también profundamente discutido, en tanto no dejaba de lado supuestos fuertemente arraigados en las representaciones de los investigadores de origen occidental. Así, acordamos con la investigadora brasilera Claudia Fonseca, quien al realizar un estado de la cuestión sobre las discusiones en torno a la categoría "familia” en el campo de la Antropología, plantea que ha pasado a ser considerada como una noción política e históricamente situada, y ha dejado de ser, de esta forma, concebida como una “unidad natural, 'célula básica' de cualquier sociedad, e institución clave para la salud mental de todo individuo" (Fonseca, 2007, p. 16). La misma autora recupera una distinción importante sobre esta categoría, resaltando que se 
ha acordado utilizarla como 'categoría nativa', y no ya como una categoría analítica, dado el peligro que encierra de colocar a la ciencia "al servicio de las verdades conservadoras del sentido común” (Fonseca, 2007, p. 16). Esta diferenciación entre categorías sociales (o nativas) y analíticas constituye una herramienta teórico-metodológica importante dentro del enfoque etnográfico. Como argumenta Elsie Rockwell, el uso analítico de categorías sociales se justifica cuando explican con mayor riqueza las dinámicas observadas (Rockwell, 2009), en el caso que señalen diferencias indistinguibles desde la teoría; es decir, que permitan dar cuenta de mayores niveles de diferenciación. Con la categoría “familia”, sucede lo contrario: dada la polisemia del término y los fuertes supuestos existentes en torno a la misma, su uso generaliza y unifica sentidos donde puede haber una variedad de dimensiones analizables y distinguibles entre sí (Cerletti, 2010).

Al pensar en la multiplicidad de experiencias que venimos mencionando en relación a los docentes, entendemos que esta distinción teórico-metodológica puede ser utilizada en términos que enriquezcan el proceso formativo de los docentes. En un análisis en profundidad sobre las diversas dimensiones que entran en juego dentro de las propias experiencias que suelen encuadrarse como familiares, se pueden distinguir analíticamente procesos históricos -y sus huellas contemporáneas de distinta índole. Esta distinción puede también acercar una forma de entender esta diversidad en el marco de relaciones sociales de desigualdad, que van sentando las bases para la construcción de representaciones en las que prima cierta noción de normalidad (vinculada, como dijimos, al modelo de familia nuclear occidental). Esta noción enmascara las condiciones reales de existencia (de posibilidades y de preferencias) de buena parte de la población, relegando a un lugar de "deficiencia" o "anormalidad" a todo lo que no se encuadra en ella. Como planteara Pierre Bourdieu, "siempre que se utilizan conceptos clasificatorios como el de familia, estos comprenden a la vez una descripción y una prescripción, que no aparece como tal porque se la acepta (casi) universalmente, y admitida como normal” (1993, p. 33).

Poner en juego la enorme diversidad y multiplicidad de experiencias vividas, en sus articulaciones con los procesos de mayor generalidad (en términos históricos) enriquece las posibilidades de abordar con complejidad y realismo una temática tan difícil como la de la "familia”, que en nuestra sociedad condensa significados y experiencias de profunda implicancia subjetiva (y objetiva) para los sujetos. Pensamos también que un proceso analítico en profundidad de este tipo, puede generar asi- 
mismo condiciones favorables para revisar los supuestos fuertemente arraigados, de sesgo negativo, que se registran recurrentemente entre los docentes respecto a las familias de sus alumnos. Recuperar el valor analítico de las propias experiencias, pueda tal vez generar una herramienta más abierta para hacer lugar a otras experiencias vividas como alejadas de lo "correcto”, de lo “bien constituido”, y quizá también para entenderlas como parte de procesos de mayor alcance, en los que se pueda pensar tanto en términos de las limitaciones estructurales en las que los sujetos viven sus vidas, como de las posibilidades y preferencias con las que se agencian los propios recorridos.

\section{Reflexiones finales}

Los sujetos construyen el propio lugar docente y el trabajo de enseñanza a través de procesos complejos que conjugan una multiplicidad de experiencias. Pero estas experiencias no siempre son visibilizadas, es decir, no se les suele atribuir mayor trascendencia al tratar la formación docente, o incluso, no son legitimadas como formas válidas de consolidar diversos conocimientos. Esto sucede muy particularmente respecto a las experiencias tematizadas como familiares, poniendo en evidencia el fuerte arraigo de supuestos prescriptivos, que muchas veces obturan una consideración más abierta sobre la diversidad social existente (ya sea tanto en términos pretéritos como presentes).

Nuestra propuesta es darle un lugar destacado a estas experiencias por el potencial que tienen para explicitar saberes y generar posibilidades de conocimientos más profundos, más enriquecidos, en el trabajo de enseñanza, y de revisar supuestos prescriptivos, muy anclados en las representaciones sobre el "deber ser". Se trata de un camino emprendido por algunos docentes e investigadores, que pensamos tiene todavía mucho por aportar.

En este sentido, entendemos que la Antropología puede hacer un aporte clave tanto para evidenciar la existencia de estas experiencias, como para conceptualizarlas, recuperando y potenciando los saberes implicados en las mismas.

Específicamente, el enfoque etnográfico permite articular contribuciones en esta dirección, por los conocimientos que produce como campo conceptual sobre los seres humanos, y las posibilidades que abre este conocimiento al poner en uso las propias experiencias -y los saberes, conscientes o inconscientes, que generan- para enriquecer la formación de los docentes y para reflexionar (y concretar) el trabajo de 
enseñanza. Como dijimos al principio, retomando el planteo de E. Rockwell (2009), permite ubicar la dimensión de lo particular, de lo local, en un marco conceptual de mayor generalidad y en sus relaciones socio-históricas más amplias.

Asimismo, es importante señalar que como todo proceso de construcción de conocimientos, estos aportes remiten a discusiones en movimiento, a dinámicas de pensamiento y debates colectivos. Justamente, pensamos que recuperar las experiencias de los sujetos, dándoles una relevancia explícita para reflexionar y ahondar sobre los propios procesos formativos, en términos que superen las categorías que prescriben más de lo que analizan, constituye una herramienta importante para reforzar un trabajo de enseñanza centrado en el desarrollo de debates de relevancia socio-histórica, con su respectivo anclaje en las subjetividades. Finalmente, entendemos que es el trabajo de discusión y problematización permanente, de apertura de conceptos, y no de cierre de definiciones, el que puede contribuir al conocimiento en profundidad tanto de los investigadores, como de los docentes y los estudiantes, hacia la complejidad y diversidad existente en la sociedad. 


\section{Referencias bibliográficas}

ACHILLI, E. Investigación y formación docente. Rosario: Laborde, 2000. 92 p.

ACHILLI, E. Investigar en Antropología Social. Los desafíos de transmitir un oficio. Rosario: Laborde, 2005. 98 p.

ALLIAUD, A. La biografía escolar en el desempeño de los docentes. Buenos Aires, Argentina: Universidad de San Andrés, n. 22, 2007. Serie Documentos de Trabajo.

BATALLÁN, G.; GARCíA, J. Antropología y Participación. Contribución al debate metodológico. Publicar en Antropología y Ciencias Sociales, n.1, p. 79-93, 1992.

BATALLÁN, G. Docentes de infancia. Antropología del trabajo en la escuela primaria. Buenos Aires: Paidós, 2007. 198 p.

BERTELEY BUSQUETS, M. La etnografía en la formación de enseñantes. Teoría Educativa, España, n. 13, p. 137-160, 2001.

BOURDIEU, P. A propos de la famille comme catégorie réalisée. Actes de la recherche en sciences sociales, n.100, p. 32-36, 1993.

BRUNER, E. Experience and its Expressions. In: TURNER, V.; BRUNER, E. (Ed.).The Anthropology of Experience, Urbana and Chicago. Champaign, IL: University of Illinois Press, 1986.p. 3-32.

CERLETTI, L. Las familias, un problema escolar? Buenos Aires: Noveduc, 2006.128 p.

CERLETTI, L. Una etnografía sobre las relaciones entre las familias y las escuelas en contextos de desigualdad social. 2010. Tesis.(Doctorado en Antropología Social) - Facultad de Filosofía y Letras, Universidad de Buenos Aires.

DE CERTEAU, M. La invención de lo cotidiano I. Artes de Hacer. México: Universidad Iberoamericana, 1996. $233 \mathrm{p}$.

EDELSTEIN, G. Problematizar las prácticas de enseñanza. Perspectiva, Florianópolis, v. 20, n. 02, p. 467-482, jul./dic., 2002.

EZPELETA, J.; ROCKWELL, E. La escuela, relato de un proceso de construcción inconcluso. México: Centro de Investigación y de Estudios Avanzados del Instituto Politécnico Nacional,1987. Mimeo.

FONSECA, C. Apresentação. De família, reprodução e parentesco: algumas considerações. Cadernos Pagu, n. 29, p. 9-35, jul./dez. 2007.

GOODSON, I. Hacia un desarrollo de las historias personales y profesionales de los docentes. Revista Mexicana de Investigación Educativa, v. 8, n.19, p. 733-758, sept./ dic. 2003. 
HELLER, A. La estructura de la vida cotidiana. In: HELLER, A. Historia y vida cotidiana. Aportación a la sociología socialista. Barcelona: Grijalbo, 1972. p. 39-69.

NEUFELD, M. R. Acerca de Antropología Social e Historia: una mirada desde la Antropología de la Educación. Cuadernos Del Instituto Nacional de Antropología y Pensamiento Latinoamericano, n.17, p. 145-158, 1996/1997.

NOGUEIRA, M. A. A categoria “família” na pesquisa em sociologia da educação: notas preliminares sobre um processo de desenvolvimento. Inter-legere, n. 9, p. 156-166, jul./dez. 2011.

ROCKWELL, E. De huellas, bardas y veredas: una historia cotidiana en la escuela. In: ROCKWELL, E. (Coord.). La escuela cotidiana. México: Fondo de Cultura Económica, 1995. p. $13-57$.

ROCKWELL, E. La Experiencia Etnográfica. Historia y cultura en los procesos educativos. Buenos Aires: Paidós, 2009. 222p.

SEGALEN, M. Antropología histórica de la familia. Madrid:Taurus, 1992. 264 p.

SINISI, L.; CERLETTI, L.; RÙA, M. La enseñanza de la Antropología y la formación de profesores. Revista Espacios de crítica y producción, n. 46, p. 94-101, sept. 2011.

Submetido à publicação em 10 de janeiro de 2013.

Aprovado em 08 de março de 2013. 